\title{
Local structural evolution in the anionic solid solution $\mathrm{ZnSe}_{x} \mathrm{~S}_{1-x}$
}

\author{
Tinku Dan, ${ }^{1}$ Ashutosh Mohanty, ${ }^{1}$ Anirban Dutta $\odot,{ }^{1}$ Rahul Mahavir Varma, ${ }^{1}$ Sagar Sarkar, ${ }^{2}$ Igor Di Marco $\odot, 2,3,4$ \\ Olle Eriksson, ${ }^{3,5}$ Edmund Welter, ${ }^{6}$ Simone Pollastri, ${ }^{7}$ Luca Olivi, ${ }^{7}$ K. R. Priolkar $\odot,{ }^{8}$ and D. D. Sarma $\odot^{1, *}$ \\ ${ }^{1}$ Solid State and Structural Chemistry Unit, Indian Institute of Science, Bengaluru 560012, India \\ ${ }^{2}$ Asia Pacific Center for Theoretical Physics, Pohang 37673, Korea \\ ${ }^{3}$ Department of Physics and Astronomy, Uppsala University, Box 516, SE-75120 Uppsala, Sweden \\ ${ }^{4}$ Department of Physics, POSTECH, Pohang 37673, Korea \\ ${ }^{5}$ School of Science and Technology, Örebro University, SE-70182 Örebro, Sweden \\ ${ }^{6}$ Deutsches Elektronen-Synchrotron, A Research Centre of the Helmholtz. Association, Notkestrasse 85, 22607 Hamburg, Germany \\ ${ }^{7}$ Elettra-Sincrotrone Trieste S.C.p.A., SS14, km 163.5 in AREA Science Park, 34149 Basovizza, Trieste, Italy \\ ${ }^{8}$ Department of Physics, Goa University, Goa 403206, India
}

(Received 26 August 2021; revised 4 November 2021; accepted 5 November 2021; published 19 November 2021)

\begin{abstract}
The century-old Vegard's law has been remarkably accurate in describing the evolution of the lattice parameters of almost all solid solutions. Contractions or expansions of lattice parameters of such systems depend on the size of the guest atom being smaller or larger than the host atom it replaces to form the solid solution. This has given rise to the concept of "chemical pressure" in analogy to the physical pressure. We have investigated using EXAFS the evolution of the local structure in terms of atom-pair distances extending up to the third-nearest neighbors in the family of compounds, $\mathrm{ZnSe}_{x} \mathrm{~S}_{1-x}$ as an example of an anionic solid solution, in contrast to all previous studies focusing on cationic solid solutions. Our results establish several common features between these two types of solid solutions, while strongly suggesting that the concept of a chemical pressure is inaccurate and misleading. Most interestingly, we also find a qualitative difference between the cationic solid solutions, reported earlier, and the anionic solid solution.
\end{abstract}

DOI: 10.1103/PhysRevB.104.184113

\section{INTRODUCTION}

It is a standard procedure in material science and engineering to alter material properties and to design materials for specific purposes by synthesizing different alloys. One of the earliest investigations into understanding the underlying structural changes that can be brought about by such alloying was by Vegard [1], who pointed out almost exactly a hundred years ago that alloying has systematic effects on the lattice parameters. Considering the two end-member compounds that define a given alloy, he showed that the lattice parameters of any composition of the alloy can be expressed as an average of the lattice parameters [2-11] of the end members weighted by the respective compositions. There have been numerous investigations that have established Vegard's law beyond any doubt. Of course, there are cases where small deviations have been observed [12-20] but these deviations are typically in the order of a few percent, thereby showing the significance and the universal validity of Vegard's law. This is a surprising result because the two end members can have vastly different lattice parameters reflecting vastly different bond lengths at the atomic level.

The impact of Vegard's law has been so impressive that the concept has been extended to suggest smooth variations of various properties and not just the lattice parameters

\footnotetext{
*sarma@iisc.ac.in
}

[3-6,8-10] between the end members of any alloy system. For example, in semiconductor physics, this concept has been used to show that the band gap of an alloy semiconductor tends to evolve approximately linearly with the composition, between the values determined by the two end-member semiconductors, for example GaAs and InAs [21]. There are many other properties that have been expressed in terms of Vegard's law, indicating that there must be some microscopic justification for its validity. Among other factors, the lattice parameters of any material or compound are controlled by the interatomic bond lengths within the unit cell, thereby suggesting that the linear variation of the lattice parameters of alloys with their composition may be a consequence of a linear variation in the interatomic bond distances. Similar thinking has often led the community to equate changing lattice parameters under the application of a physical pressure to changing lattice parameters via alloying, labeling the latter process as chemical pressure. Intriguingly, however, over the years several experiments $[8,22-24]$ on the extended $\mathrm{x}$-ray absorption fine structure (EXAFS) have established a relative invariance of individual bond lengths in alloy systems. This has cast severe doubts on the extensively used concept of chemical pressure [23].

In Ref. [23], the $\mathrm{Zn}_{x} \mathrm{Cd}_{1-x} \mathrm{~S}$ alloy with $0 \leqslant x \leqslant 1$ was investigated to understand the relation between Vegard's law and bond lengths. While the nearest neighbors (1st coordination shell) were found to be relatively invariant, the interatomic distances of the $\mathrm{Cd}-\mathrm{Cd}$ and $\mathrm{Zn}-\mathrm{Zn}$ pairs (2nd 
coordination shell) were shown to change much more rapidly, but still falling short of explaining the observed changes in the lattice parameters with respect to the alloy composition. These changes, instead, were found to be accounted for by the rapid changes of the $\mathrm{Cd}-\mathrm{S}$ and $\mathrm{Zn}-\mathrm{S}$ interatomic distances (3rd coordination shell). All these observations could be explained primarily in terms of changes in the $M-\mathrm{S}-M$ bond angles with $M=\mathrm{Cd}$ or $\mathrm{Zn}$ along with relative invariances of the nearest-neighbor $M-S$ bond distances. Overall, the emerging microscopic description of the structural evolution in alloys seems to be fundamentally different from the application of a physical pressure where individual bond distances change proportionately with the lattice parameters. This suggests that the association of the physical and the so-called "chemical" pressure is a derivative of a misplaced interpretation of Vegard's law, where the macroscopically averaged lattice constants measured by diffraction techniques are presumed to control linearly the microscopic bond distances.

Interestingly, EXAFS studies on such solid solutions in inorganic compounds have been exclusively focused on cationic substitutions, for example $\mathrm{Zn}_{x} \mathrm{Cd}_{1-x} \mathrm{~S}$ [23], $\mathrm{Ga}_{x} \mathrm{In}_{1-x}$ As [22], $\mathrm{Mn}_{x} \mathrm{Cd}_{1-x} \mathrm{Te}$ [8], or $\mathrm{YIn}_{x} \mathrm{Mn}_{1-x} \mathrm{O}_{3}$ [24]. While anionic substitution is also a well-established route to tune material properties, there is no information available in the literature on the microscopic evolution of various atom-pair distances in an anionic-substituted inorganic system despite the known validity of Vegard's law for such systems. In the present study, we shall fill this gap by investigating the atom-pair distances in $\mathrm{ZnSe}_{x} \mathrm{~S}_{1-x}$, where $0 \leqslant x \leqslant 1$. Our analysis is based on the EXAFS measured at the $\mathrm{Zn}, \mathrm{Se}$, and $\mathrm{S}$ K-edges, in conjunction with $a b$ initio electronic structure calculations with full geometry optimizations. Based on a quantitatively different microscopic evolution of the structural motifs in this system compared to the earlier investigated cationic substituted systems, this study provides a complete insight into the microscopic structural evolution in solid solution systems independent of the site of the substitution.

\section{EXPERIMENTAL DETAILS AND DATA ANALYSIS}

$\mathrm{ZnSe}_{x} \mathrm{~S}_{1-x}$ samples with $x=1,0.9,0.8,0.7,0.5$, and 0.0 were synthesized by the solid-state method, starting with the desired stoichiometric ratio of $\mathrm{ZnSe}$ and $\mathrm{ZnS}$. The component powders were thoroughly mixed and then pelletized. The pellet was sealed in a quartz ampule at a vacuum of $2 \times$ $10^{-6}$ mbar. This quartz ampule containing the pellet was then heated in the furnace at $580{ }^{\circ} \mathrm{C}$ for 36 hours with a slow heating and cooling protocol. X-ray diffraction (XRD) was used to determine the phase purity of the prepared samples. The grinding, pelletizing, sealing, and heating cycles were continued till no impurity phase was detected in XRD. EXAFS data at the $\mathrm{Zn}, \mathrm{Se}$, and $\mathrm{S} \mathrm{K}$-edges was recorded in transmission. Fluorescence mode of detection was used for S K-edges in compounds with $x=0.9,0.8$ and 0.7. $\mathrm{Zn}$ and Se K-edge EXAFS measurements at room temperature and $30 \mathrm{~K}$ were performed at P65 beamline [25] at PETRA III synchrotron, DESY, Hamburg. For these measurements the absorbers were prepared using the layered scotch tape method. The thickness of the absorber was adjusted such that the absorption edge jump $\Delta \mu t \leqslant 1$. For the $\mathrm{S} \mathrm{K}$-edge measurements, the required quantity of sample was spread on a cellular membrane with the help of a solvent. Powder samples were mixed with water and were precipitated on a cellular membrane using a vacuum pressure set up. The $\mathrm{S}$ K-edge EXAFS data were collected at $300 \mathrm{~K}$ at the XAFS beamline at Elettra synchrotron, Trieste [26]. Results obtained from $\mathrm{Zn}$ and Se K-edges with 30 and $300 \mathrm{~K}$ were consistent with each other with a reduced thermal contribution for the $30 \mathrm{~K}$ data. Therefore, all data and analyses presented in this manuscript is from the $\mathrm{Zn}$ and Se K-edge data obtained with the sample at $30 \mathrm{~K}$. However, the S K-edge data could only be collected at room temperature and presented here.

Data analyses were carried out using Demeter suite [27], which uses FEFF 6.0 code [28]. The EXAFS $\chi(k)$ oscillations at the $\mathrm{Zn}, \mathrm{Se}$, and $\mathrm{S} \mathrm{K}$-edges were obtained by individually subtracting the pre-edge background, normalizing to the experimental edge step, and subtracting a smooth atomic background from the normalized absorption data using the Athena program. The resulting $\chi(k)$ were fitted to a model based on the crystal structure using the Artemis program [27]. Detailed fittings were carried out using the structural parameters corresponding to the zinc blende structure appropriate for these compounds, focusing on EXAFS data in the k range extending over $3-15 \AA^{-1}$ for $\mathrm{Zn}-\mathrm{K}$ and Se-K edges, and 3-12 $\AA^{-1}$ for S-K edge. The inverse Fourier transform was performed for the $R$ range between 1.00 and $4.76 \AA$. We fit the EXAFS data from $\mathrm{Zn}$ and Se K-edges simultaneously within a single structural model that automatically imposes these constraints on $\mathrm{Zn}-\mathrm{Se}$ bond distances for the first and the third-nearest neighbors in order to obtain the most robust information on the local structures in all cases. Complete details of EXAFS data analyses can be found in the Supplemental Material (SM) [29]. The contributions of near neighbor scattering paths to the EXAFS signal were computed using the FEFF code based on $a b$ initio selfconsistent real space multiple-scattering calculations [28] and implemented in Artemis software. Various bond distances $(R)$, mean-square-displacement in these distances $\left(\sigma^{2}\right)$, amplitude reduction factors $\left(S_{0}^{2}\right)$, and corrections to edge energies $\left(\Delta E_{0}\right)$ were allowed to vary during the fitting. However, the coordination number $(N)$ for each scattering path was kept constant.

\section{COMPUTATIONAL DETAILS}

Electronic structure calculations were performed for the $\mathrm{ZnSe}_{x} \mathrm{~S}_{1-x}$ alloy with $x=1.00,0.75,0.50,0.25$, and 0.00 , by means of a projected augmented wave (PAW) [30] implementation of density functional theory (DFT) within the Vienna ab initio simulation package (VASP) [31-34]. The generalized gradient approximation (GGA) [35] in the formulation by Perdew-Burke-Ernzerhof (PBE) [36] was used for the exchange-correlation functional. The experimental zinc blende structure (space group F-43m) was used as a starting point for the structural optimization of both $\mathrm{ZnS}$ and $\mathrm{ZnSe}$ [37]. For the other three compositions investigated, namely $\mathrm{ZnS}_{0.75} \mathrm{Se}_{0.25}, \mathrm{ZnS}_{0.50} \mathrm{Se}_{0.50}$, and $\mathrm{ZnS}_{0.25} \mathrm{Se}_{0.75}$, disorder was analysed by means of special quasirandom structures (SQS) [38]. The latter were generated using a Monte Carlo simulation based method [39] as implemented in the alloy theoretic automated toolkit (ATAT) [40-42]. Convergence in the 
distribution of the bond lengths and bond angles with respect to the number of atoms in the supercell was checked by doing the calculations with 64, 96, and 128 atom supercells respectively. Hence, the physical properties of the $\mathrm{ZnSe}_{x} \mathrm{~S}_{1-x}$ alloy are directly extracted from the calculations of the 128 atom supercell. In more quantitative terms, the pair correlation function in the 128 atom supercell matches to that of a perfectly random alloy until the $7^{\text {th }}$ coordination shell. The equilibrium structures were determined by optimizing internal positions and lattice parameters till the forces on the atoms were smaller than $10^{-3} \mathrm{eV} / \AA$. The integrals over the Brillouin Zone were performed by means of a $\Gamma$-centered Monkhorst-Pack k mesh of $14 \times 14 \times 14$ for pristine $\mathrm{ZnS}$ and $\mathrm{ZnSe}$, while a k-mesh of $10 \times 10 \times 5$ was used for the 128 atom supercells. An energy cutoff of $650 \mathrm{eV}$ was used for the kinetic energy of the plane waves included in the basis. Convergence of the full structural optimization with respect to plane wave energy cutoff and $\mathbf{k}$ mesh was checked for all systems. To understand the quantitative precision of our calculations, we can compare the optimized lattice parameter for $\mathrm{ZnS}$ to the average of reported experimental values [37]. The theoretical value of $5.45 \AA$ is $0.70 \%$ larger than the experimental reference, which we attribute to the general overestimation associated with PBE. Hence, we conclude that our theoretical analysis is very accurate, qualitatively as well as quantitatively.

\section{RESULTS AND DISCUSSION}

In Fig. 1, we show the x-ray diffraction patterns of $\mathrm{ZnSe}_{x} \mathrm{~S}_{1-x}$ for various $x$ values. We have also shown for comparison the standard diffraction patterns of $\mathrm{ZnSe}$ and $\mathrm{ZnS}$ from the ICSD data base. These XRD patterns show that these compounds form in the cubic zinc blende structure. With an increasing incorporation of larger Se anions at the $\mathrm{S}$ sites, one finds a systematic variation of lattice parameters to larger values indicated by the progressive movement of the diffraction peaks to lower angles in Fig. 1(a), illustrated by the comparison of the peak positions relative to the verticaldashed line at $45^{\circ}$. We have extracted the lattice parameter from the XRD pattern for each composition and plotted it in Fig. 1(b) as a function of the Se content $x$. The intermediate lattice parameters show a linear variation between the two end members, as indicated by the red-dashed line. This linear variation with the composition is a confirmation of the fact that this system obeys the Vegard's law.

Calculated lattice parameters obtained from geometry optimized calculations for each composition $(x=0.0,0.25,0.5$, 0.75 , and 1.0) are also shown in Fig. 1(b) with blue open circles with the corresponding y-axis for the lattice parameters shown on the right. We note that the right $y$-axis values are slightly shifted with respect to those on the left axis to take into account a small mismatch between the calculated and the experimental values of lattice parameters with the calculated values being slightly larger than the experimental ones. However, the relative scales on both axes are the same allowing us a direct comparison of changes in the lattice parameter with the composition obtained from calculations with the changes observed experimentally. We find a good agreement between the experiment and calculation, confirming that the theoretical
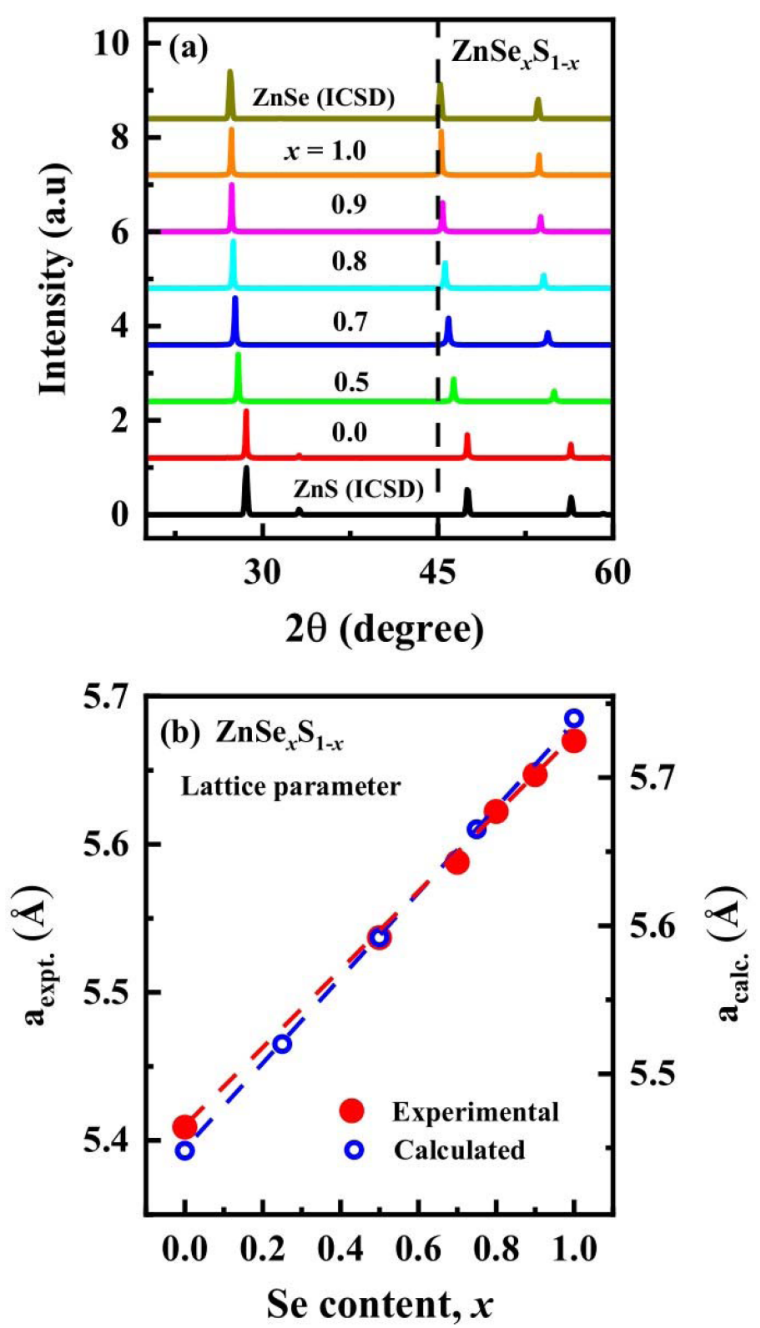

FIG. 1. (a) X-Ray diffraction (XRD) patterns of $\mathrm{ZnSe}_{x} \mathrm{~S}_{1-x}$ solid solution for different values of $x$. The peaks shift systematically towards lower $2 \theta$ values with the increase in selenium concentration $(x)$ as can be seen with the help of the vertical dashed reference line at $45^{\circ}$. (b) The lattice parameter $a$ (in $\AA$ ) from experiment (red-solid circles) and calculations (blue-open circles). The experimental points are obtained from Rietveld analyses of the diffraction patterns as a function of the composition $x$.

methods employed here capture the essential aspects of the evolution of lattice parameters with the composition. This then allows us to extract other measurable quantities from these calculations to make a more direct comparison with estimates obtained from the EXAFS experiments.

The $k^{2}$-weighted EXAFS oscillations as a function of $k$ for all compositions $x$ are shown in Figs. 2(a), 2(b), and 2(c) for Zn K-edge, Se K-edge, and S K-edge, respectively. Prominent oscillations with good signal-to-noise ratios are seen for $k \leqslant$ $13 \AA^{-1}$ for all edges. We show the corresponding Fourier transforms of Zn K-edge, Se K-edge, and S K-edge EXAFS data in Figs. 2(d), 2(e), and 2(f), respectively. These figures show significant changes with respect to the alloy composition and provide us with some understanding of the evolution of the local structure as a function of $x$ in this solid solution. Figure 2(d) shows two main groups of features; one is a single 

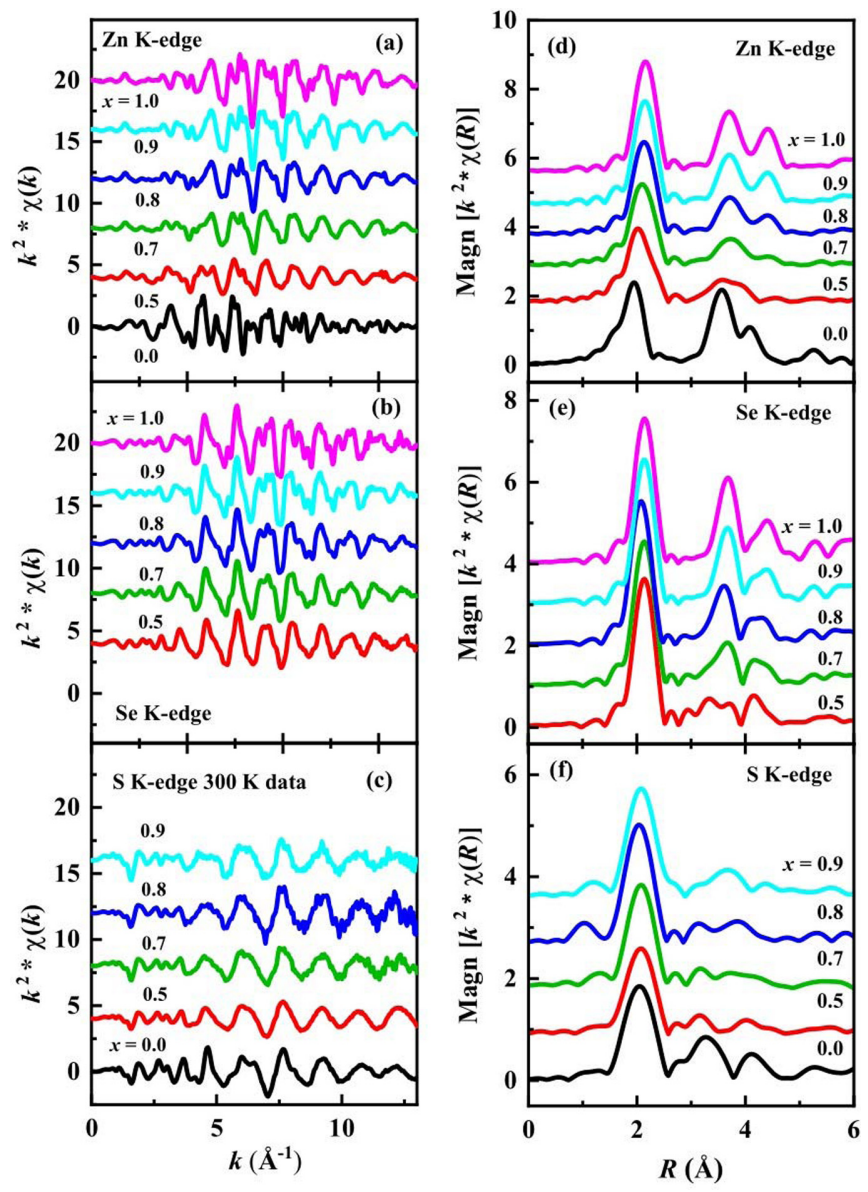

FIG. 2. $k^{2}$-weighted EXAFS oscillations obtained at (a) the $\mathrm{Zn}$ K-edge, (b) Se K-edge, and (c) S K-edge for different compositions (x) of $\mathrm{ZnSe}_{x} \mathrm{~S}_{1-x}$. The corresponding Fourier transforms are shown for the Zn K-edge (d), Se K-edge (e), and S K-edge (f). Details of data analysis are given in the Supplemental Material [29].

peak-like feature around $2 \AA$, while the other is a doublet-like feature around 3-5 $\AA$. The first peak around $2 \AA$ arises from the scattering from the nearest-neighbor coordination shell, $\mathrm{Zn}-\mathrm{S}$ or $\mathrm{Zn}-\mathrm{Se}$. The scattering peak moves systematically to higher distances with an increasing Se content, which reflects a longer $\mathrm{Zn}$-Se bond. The farther features appearing between 3 and $5 \AA$ arise from the 2 nd and the 3rd coordination shells of these compounds. Major contributions are due to the $\mathrm{Zn}-$ $\mathrm{Zn}$ scattering from the second shell and to $\mathrm{Zn}-\mathrm{S}$ or $\mathrm{Zn}-\mathrm{Se}$ scattering from the third shell. The well-defined peak arising from the $\mathrm{Zn}-\mathrm{Zn}$ scattering shows a systematic increase across the composition series. Interestingly, the Fourier transforms reported in Figs. 2(e) and 2(f) do not show any appreciable shift in the peak positions with the composition. Since the first peak appearing near $2 \AA$ corresponds to Se-Zn scattering in panel (e) and S-Zn scattering in panel (f), these results suggest an invariance of these bond distances with the composition, despite a systematic change in the lattice parameter. The peak positions in Figs. 2(d), 2(e), and 2(f), however, cannot be directly interpreted as the bond distances because of the various scattering phase shifts that make the peaks appear at a distance different from the actual bond distances.

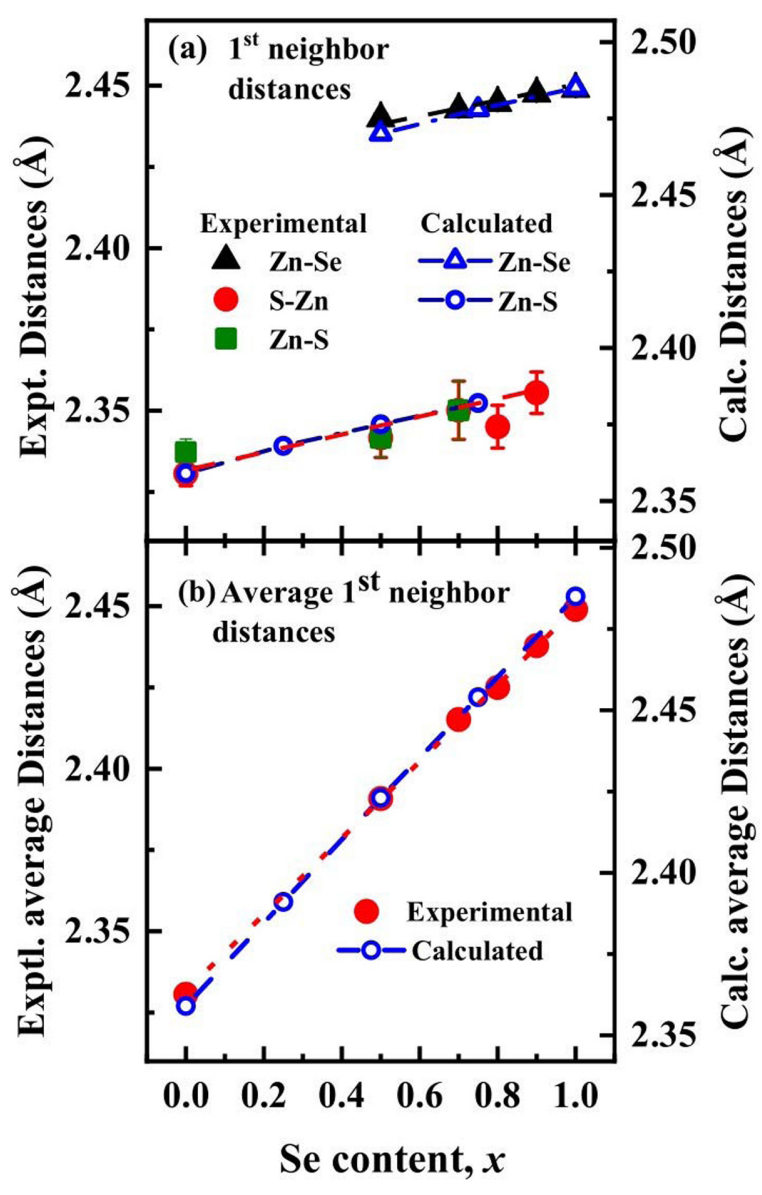

FIG. 3. (a) Experimentally obtained bond distances: Zn-Se (black-solid triangles) and $\mathrm{Zn}-\mathrm{S}$ (green-solid squares from $\mathrm{Zn} \mathrm{K}$ edge and red-solid circles from $\mathrm{S}$ K-edge analyses) belonging to the first coordination shell as a function of the composition along with the calculated bond distances shown with corresponding blue-open symbols. (b) Composition averaged nearest-neighbor bond distances (red-solid circles from experimental estimates and blue-open circles from the calculations) as a function of the composition, showing a close adherence of the averaged cation-anion bond distance to the expectation based on the Vegard's law.

We now turn to the quantitative analyses of the EXAFS results in order to obtain a detailed description of the evolution of the local structures as a function of the composition in $\mathrm{ZnSe}_{x} \mathrm{~S}_{1-x}$. While all derived parameter values from the EXAFS analysis are presented in tables in the SM, here we show plots of various interatomic distances as a function of the composition separately presenting atom-pairs belonging to different coordination shells. This provides us an effective insight into the evolution of the local structure with composition around the central atom where the EXAFS data is collected from. Figure 3(a) shows three sets of nearest-neighbor bond lengths separately obtained from the analysis of the EXAFS data. $\mathrm{Zn}-\mathrm{Se}$ bond lengths, shown by black-solid triangles, are obtained from simultaneous, constrained fitting of EXAFS at the $\mathrm{Zn}$ and Se K-edges, as discussed before. The red-solid circles show the $\mathrm{S}-\mathrm{Zn}$ bond lengths obtained from the analyses of the $\mathrm{S}$ K-edges, while the green-solid squares show the same $\mathrm{Zn}-\mathrm{S}$ bond lengths but obtained from an analysis of the $\mathrm{Zn}$ 
K-edge EXAFS data. Ideally, these two bond distances should be the same as they describe the same $\mathrm{Zn}-\mathrm{S}$ bond length. However, we present these separately, since these two, derived from two very different measurements, provide us with independent measures of the bond length, the difference between the two measures serving as an estimate of the reliability of the technique. In the highly magnified scale of Fig. 3(a), both $\mathrm{Zn}-\mathrm{Se}$ and $\mathrm{Zn}-\mathrm{S}$ bond lengths show an approximately linear increase with Se content, as emphasized by the dashed straight lines drawn through the data points as a guide to the eye.

It can be argued that the increase in the $\mathrm{Zn}-\mathrm{S}$ bond length reflects a negative chemical pressure around the $\mathrm{Zn}$ site. This is due to the substitution of $\mathrm{S}$ with a larger ion, Se, thereby increasing the lattice parameters and the $\mathrm{Zn}-\mathrm{S}$ bond length. If we consider the $\mathrm{ZnSe}$ rich end of the composition, instead, replacing Se with $\mathrm{S}$ leads to a contraction of the $\mathrm{Zn}-\mathrm{Se}$ bond lengths. This reflects a positive chemical pressure on the system, brought about by a doping with the smaller $\mathrm{S}$ ions at the larger Se sites. A more insightful analysis can be made by comparing the percentage change of these bond lengths $(\xi)$ with respect to a unit change in the composition, which is the value of $x$ in $\mathrm{ZnSe}_{x} \mathrm{~S}_{1-x}$. From Fig. 3(a), we estimate $\xi$ to be about $1.2 \%$ and $0.7 \%$ for the $\mathrm{Zn}-\mathrm{S}$ and $\mathrm{Zn}-\mathrm{Se}$ bonds, respectively. If these bond lengths were to change according to Vegard's law, there would be a single fictitious bond whose length would linearly interpolate from the value of $\mathrm{Zn}-\mathrm{S}$ bond length in $\mathrm{ZnS}$ to that of $\mathrm{Zn}-\mathrm{Se}$ at the other end; we can calculate the corresponding idealized rate of change of this fictitious bond, labeled as $\xi_{\text {vegard }}$. In the present case, for the first coordination shell, we estimate $\xi_{\text {vegard }}=5.1 \%$, which is very different from the values extracted from the experiment. This analysis demonstrates that the $\mathrm{Zn}-\mathrm{S}$ and $\mathrm{Zn}-\mathrm{Se}$ bonds are much more rigid than what one could expect from Vegard's law. This is very similar to what was reported for $\mathrm{Zn}_{x} \mathrm{Cd}_{1-x} \mathrm{~S}$ solid solutions, where the $\mathrm{Zn}-\mathrm{S}$ and $\mathrm{Cd}-\mathrm{S}$ bond distances are found to change much less than what is predicted by Vegard's law [23].

Overall, the changes in the nearest-neighbor bond lengths in both cationic and anionic substituted systems do not reflect the changes observed in the lattice parameter with varying compositions. At this stage, we must stress that the application of physical pressure to any given compound would usually lead to changes in the lattice parameter in correspondence to those of the nearest-neighbor bond lengths in absence of any phase transition [43]. Therefore, drawing an analogy between composition-driven and pressure-driven changes in the lattice parameters does not appear to be very accurate. In other words, the concept of "chemical pressure", extensively used in tuning properties of various systems, is at best a misnomer, arising from the limitation of the Vegard's law, suitable for describing lattice parameters, in providing a description of the microscopic evolution of the local structural aspects in terms of the interatomic bond distances.

In order to obtain a microscopic understanding of these results, we have performed extensive large-scale $a b$ initio calculations for a series of $\mathrm{ZnSe}_{x} \mathrm{~S}_{1-x}$ compositions, namely for $x=0.00,0.25,0.50,0.75$, and 1.00 , as discussed in detail in the methodology section. The final geometry of the optimized structure for each composition was analyzed for extracting the nearest-neighbor $\mathrm{Zn}-\mathrm{S}$ and $\mathrm{Zn}-\mathrm{Se}$ bond lengths. These bond lengths are found to depend on the local chemical environment, giving rise to a distribution of nearest-neighbor bond lengths. The average values of the distributions are shown in Fig. 3(a), where blue-open triangles and circles are used for $\mathrm{Zn}-\mathrm{Se}$ and $\mathrm{Zn}-\mathrm{S}$, respectively. The slight mismatch of the calculated lattice parameters with those obtained from experiments [see Fig. 1(b)] translates into a similar mismatch between the calculated and experimental bond lengths; this is accounted for by a rigid shift of the right axis of Fig. 3(a) with respect to its left axis that brings the calculated and experimentally determined bond lengths of the end members, $\mathrm{ZnS}$ and $\mathrm{ZnSe}$, to appear on top of each other. This allows us to make a direct comparison between the rates of change of the nearest-neighbor bond lengths obtained from the calculations (dashed-blue lines) and those obtained from the experiments (dashed-black and red lines). The fact that the curves are almost on top of each other highlights the remarkable agreement between experiment and theory.

To investigate the Vegard's law in microscopic terms, we plot the nearest-neighbor bond lengths for the two end members, $\mathrm{ZnS}$ and $\mathrm{ZnSe}$, using the same symbol (red-solid circles) in Fig. 3(b). Then, we draw a dotted-red line connecting these two points, which shows the expected variation of a single, fictitious, nearest-neighbor cation-anion bond distance with the composition. This corresponds to a microscopic modeling of the solid solution in terms of a virtual crystal approximation (VCA) for the alloy system [44]. Finally, we also include the composition-weighted average of the experimentally-determined nearest-neighbor bond distances, $\mathrm{Zn}-\mathrm{S}$ and $\mathrm{Zn}-\mathrm{Se}$, for all the intermediate compositions, using again red-solid circles. Clearly, the average bond distance interpolates linearly between the two end members, in excellent agreement with dotted line connecting the end members, which is inspired by Vegard's law. The same conclusions can be drawn using the bond lengths obtained from the calculations, as shown with blue open circles in Fig. 3(b). This shows that the adherence of the composition-dependent lattice parameters to the Vegard's law arises from a very different microscopic evolution than that of the individual bond lengths, and should only be thought of in an average sense, characterised by sampling over many unit cells. Since the lattice parameters are determined from experiments that necessarily average over many unit cells to have properly defined x-ray diffraction peaks, the averaged lattice parameters obtained from these experiments have always conformed to the Vegard's law. However, EXAFS is specifically sensitive to the local structure and is devoid of any such space averaging, thereby leading to results that do not follow a Vegard's law type dependence on the composition. These results lead one to naturally ask about the length-scale of averaging that is required to arrive at the global description captured by $\mathrm{x}$-ray diffraction experiments starting from the local description obtained from EXAFS. In the following, we address this issue by determining the interatomic distances between pairs of atoms that are increasingly farther apart.

We first focus on the 2nd coordination shell, by analyzing the $\mathrm{Zn}$ K-edge data, which relate uniquely to $\mathrm{Zn}-\mathrm{Zn}$ distances. For intermediate compositions, we found it necessary to adopt a model with two different $\mathrm{Zn}-\mathrm{Zn}$ distances to obtain a satisfactory fit to the EXAFS oscillation, since no model with a 


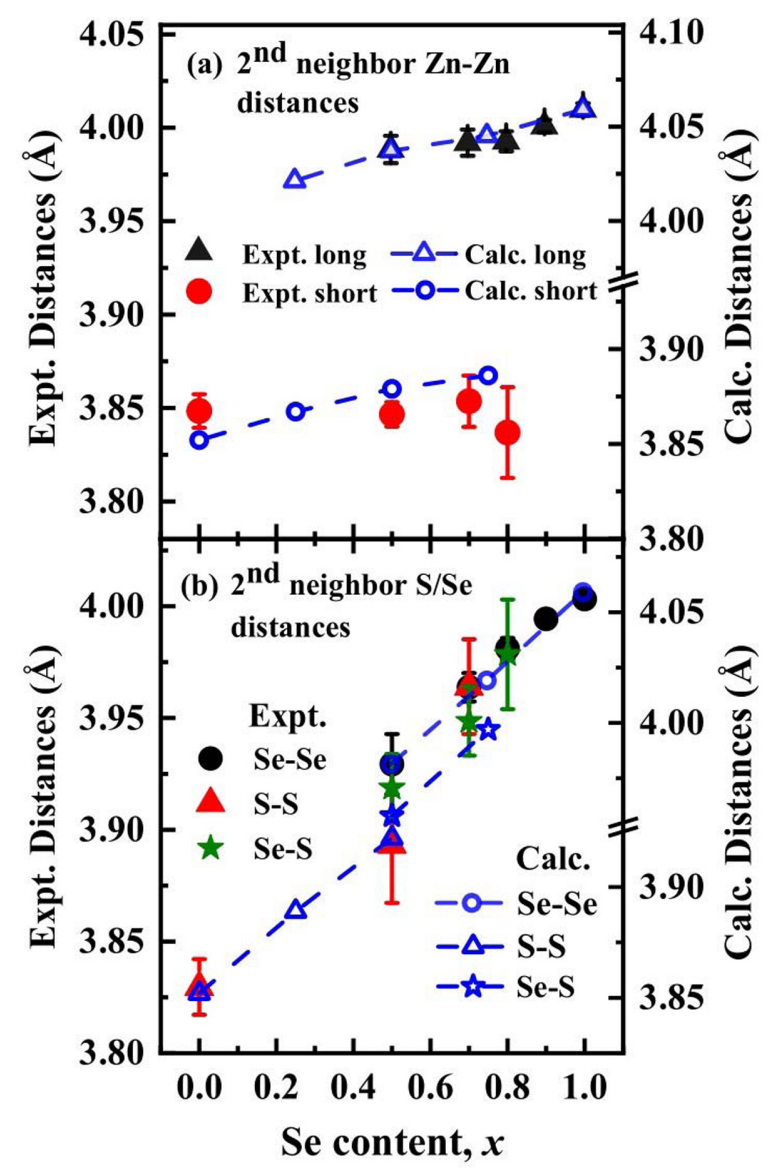

FIG. 4. (a) Experimentally obtained two Zn-Zn distances (long: black-solid triangles, short: red-solid circles) for the second coordination shell as a function of the composition along with the corresponding calculated quantities shown with blue-open symbols. (b) Experimentally obtained Se-Se (black-solid circles), S-S (redsolid triangles), and Se-S (green-solid stars) distances as a function of the composition, along with the calculated quantities with corresponding blue-open symbols.

single $\mathrm{Zn}-\mathrm{Zn}$ distance could adequately describe the experimental data. This stems from the fact that $\mathrm{Zn}-\mathrm{Zn}$ distances are defined by the $\mathrm{Zn}-\mathrm{S}-\mathrm{Zn}$ and $\mathrm{Zn}-\mathrm{Se}-\mathrm{Zn}$ connectivities. Since the bond length of $\mathrm{Zn}-\mathrm{S}$, which is the same as $\mathrm{S}-\mathrm{Zn}$, is distinctly different from that of $\mathrm{Zn}-\mathrm{Se}$ (and $\mathrm{Se}-\mathrm{Zn}$ ), it is evident that we need a shorter $\mathrm{Zn}-\mathrm{Zn}$ distance for the $\mathrm{Zn}$ pairs connected via an $\mathrm{S}$ atom and a longer $\mathrm{Zn}-\mathrm{Zn}$ distance for the $\mathrm{Zn}$ pairs bridged by an Se atom. The variations of these two $\mathrm{Zn}-\mathrm{Zn}$ distances as a function of Se content are shown in Fig. 4, using black-solid triangles for the long distance and red-solid circles for the short one. The bimodal $\mathrm{Zn}-\mathrm{Zn}$ distances can be seen to retain values close to the $\mathrm{Zn}-\mathrm{Zn}$ distances found in pure $\mathrm{ZnS}$ and $\mathrm{ZnSe}$. The small variations with respect to the alloy composition are compatible with the minor variations of the individual $\mathrm{Zn}-\mathrm{S}$ and $\mathrm{Zn}-\mathrm{Se}$ bond lengths, reported in Fig. 3. Interestingly, this is in contrast to what was reported for the closely related $\mathrm{Zn}_{x} \mathrm{Cd}_{1-x} \mathrm{~S}$ system [23]; in that case, it was found that the cation-cation second shell distances between $\mathrm{Zn}-\mathrm{Zn}, \mathrm{Zn}-\mathrm{Cd}$, and $\mathrm{Cd}-\mathrm{Cd}$ atom pairs changed more rapidly with the composition than the bond distances in the first coordination shell. This different behavior found in the second coordination shell signals, for the first time, the qualitative difference in the evolution of the local structures between a cation-substituted system $\left(\mathrm{Zn}_{x} \mathrm{Cd}_{1-x} \mathrm{~S}\right)$ and an anion-substituted system $\left(\mathrm{ZnSe}_{x} \mathrm{~S}_{1-x}\right)$. It is important to note that this unexpected finding is also confirmed by the electronic structure calculations, which show that the longer $\mathrm{Zn}-\mathrm{Zn}$ bonds indeed arise from those $\mathrm{Zn}$ pairs that are bridged by $\mathrm{Se}$ atoms while the shorter $\mathrm{Zn}-\mathrm{Zn}$ pairs are invariably connected by a bridging $S$ atom, thereby leading to a bimodal distribution. These average $\mathrm{Zn}-\mathrm{Zn}$ bond distances are plotted separately in Fig. 4(a), using blue-open triangles and circles for the Se and S bridged pairs, respectively. Clearly, the longer $\mathrm{Zn}-\mathrm{Zn}$ bonds are in remarkable agreement with the experimental data. Even the short $\mathrm{Zn}-\mathrm{Zn}$ bonds exhibit a reasonable agreement, if we take into account the larger uncertainties associated with extracting estimates of the short $\mathrm{Zn}-\mathrm{Zn}$ bonds for large values of $x(\geqslant 50)$, due to the reduced number of such pairs in the dilute $\mathrm{S}$ end of the solid solution.

The second coordination shell with the anions at the center includes Se-Se, S-S, and Se-S (or S-Se) atom pairs. The distances between them were extracted, for all compositions, by analyzing the corresponding Se and S K-edge EXAFS, and are shown in Fig. 4(b) with solid-black circles, red triangles, and green stars, respectively. The $\mathrm{S}-\mathrm{S}$ distances, extracted from the S K-edge EXAFS analysis, could be estimated only up to $x \leqslant 50$ with reasonable accuracy. Similarly, the Se-S distances could be estimated for only $x=50$ and 70 due to a low contribution from S scattering for more Se-rich compositions. Nevertheless, these limited data illustrate clearly the trends with the compositions and can be used as a further test to evaluate the accuracy of the computational model. The average $\mathrm{Se}-\mathrm{Se}, \mathrm{S}-\mathrm{S}$, and $\mathrm{Se}-\mathrm{S}$ atom pair distances extracted from the calculations are plotted in Fig. 4(b), using corresponding blue open symbols. The excellent agreement between theory and experiment confirms that the calculations can be trusted for analyzing diluted $\mathrm{S}$ compositions, where the experiment suffers from a low contribution from $\mathrm{S}$ scattering, as mentioned above. Overall, our results show a strikingly different trend for the anion-anion atom-pair distances [Fig. 4(b)] in comparison to the cation-cation atom-pair distances [Fig. 4(a)]. The anion-anion atom-pair distances exhibit a rapid variation with composition, almost in conformity with the Vegard's law, in contrast to the relatively rigid behavior of the cation-cation distances.

To understand these fundamental differences, we note that every anionic pair is bridged by a $\mathrm{Zn}$ atom, thereby leading to Se-Zn-Se, S-Zn-S, and Se-Zn-S bonds. If we keep in mind that the individual $\mathrm{Zn}-\mathrm{S}$ or $\mathrm{Zn}-\mathrm{Se}$ bond lengths do not change appreciably across the composition of the solid solution [see Fig. 3(a)], then the only way the anionic pairs can vary significantly is by changing the angles between the $\mathrm{Se}-\mathrm{Zn}-\mathrm{Se}, \mathrm{S}-\mathrm{Zn}-\mathrm{S}$, and $\mathrm{Se}-\mathrm{Zn}-\mathrm{S}$ bonds around the central $\mathrm{Zn}$ atom. In contrast, for cationic pairs, the relative invariance of $\mathrm{Zn}-\mathrm{Zn}$ atom-pair distances reflects that the $\mathrm{Zn}-\mathrm{Se}-\mathrm{Zn}$ and $\mathrm{Zn}-\mathrm{S}-\mathrm{Zn}$ bond angles around the anions (Se or $\mathrm{S}$ ) do not change much with the composition. This argument can be reformulated on a quantitative basis by calculating the average angles of the anionic and cationic pairs in the second coordination shell. We have calculated the bond angles by considering triangles formed by the bond distances reported 


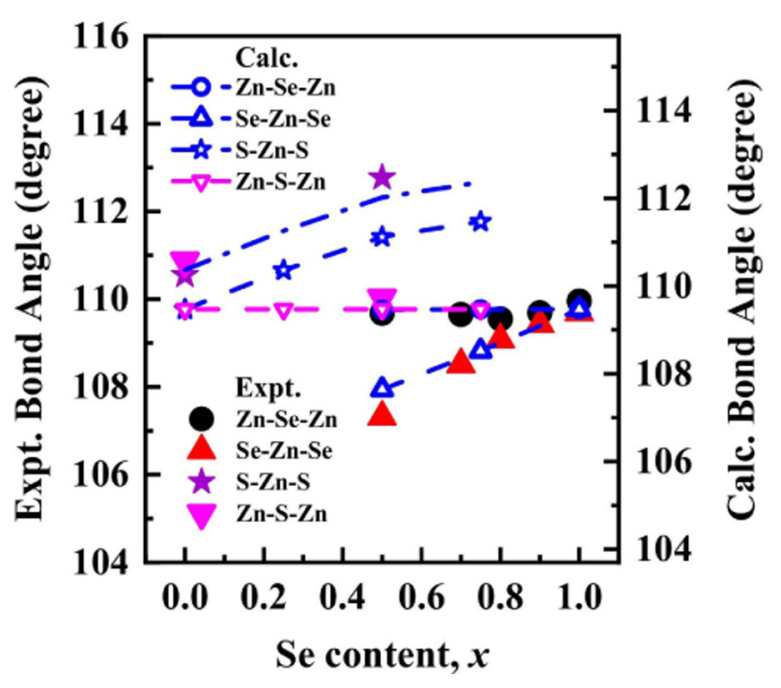

FIG. 5. Zn-Se-Zn (black-solid circles), Se-Zn-Se (red-solid up triangles), $\mathrm{S}-\mathrm{Zn}-\mathrm{S}$ (purple-solid stars), and Zn-S-Zn (magenta-solid down triangles) bond angles obtained from first and second coordination shell bond distances presented in Figs. 3(a), 4(a), and 4(b). S-Zn-S bond angles and Zn-S-Zn bond angles for dilute $\mathrm{S}$ compositions $(x \geqslant 50 \%)$ could not be estimated with any reasonable accuracy due to the large uncertainties in the S-S and short $\mathrm{Zn}-\mathrm{Zn}$ bond distances (see Fig. 4). The average angles evaluated from the calculations are also shown with corresponding blue-open symbols, indicating a very good agreement in most cases. The experimentally obtained S-Zn-S angles appear to be slightly shifted with respect to the calculated values, as shown by the dc-shifted blue dashed line; this mismatch is within the uncertainty of estimating these angles from the individual bond distances. All angles obtained for the two pure compounds are in good agreement with the tetrahedral bond angle $\left(109.47^{\circ}\right)$.

in Figs. 3(a), 4(a), and 4(b). The experimental estimates of the angles of the $\mathrm{Zn}-\mathrm{Se}-\mathrm{Zn}, \mathrm{Se}-\mathrm{Zn}-\mathrm{Se}, \mathrm{S}-\mathrm{Zn}-\mathrm{S}$, and $\mathrm{Zn}-\mathrm{S}-\mathrm{Zn}$ bonds are plotted in Fig. 5, using solid-black circles, red triangles, and purple stars, respectively. We can also extract the angles from the optimised structures obtained via DFT, which leads to a distribution of angles due to the presence of a number of symmetry-inequivalent sites in the large super-cells considered. We obtain the average over this distribution of angles to compare with the EXAFS derived angles, as shown in Fig. 5 using corresponding blue-open symbols. Theoretical and experimental data are in excellent agreement, particularly for the $\mathrm{Zn}-\mathrm{Se}-\mathrm{Zn}$ and $\mathrm{Se}-\mathrm{Zn}-\mathrm{Se}$ angles. The slight mismatch obtained for the $\mathrm{S}-\mathrm{Zn}-\mathrm{S}$ bond angles is within the expected error in the experimental estimation; this is clear from the experimental estimate of the $\mathrm{S}-\mathrm{Zn}-\mathrm{S}$ bond angle for pure $\mathrm{ZnS}$ being nearly $1^{\circ}$ higher than the known value of the tetrahedral bond angle $\left(109.47^{\circ}\right)$. From this analysis, it is evident that the increase in the lattice parameter [Fig. 1(b)] is substantially controlled by the increasing metal-centered bond angles (Fig. 5) rather than by the modest expansion of the individual $\mathrm{Zn}-\mathrm{S}$ or $\mathrm{Zn}-\mathrm{Se}$ bonds [Fig. 3(a)] and hardly by the nearly constant anion-centered bond angle (shown for $\mathrm{Zn}-\mathrm{Se}-\mathrm{Zn}$ in Fig. 5). This is in sharp contrast to what would happen under the application of a physical pressure, which would result in the change of bond lengths instead of bond

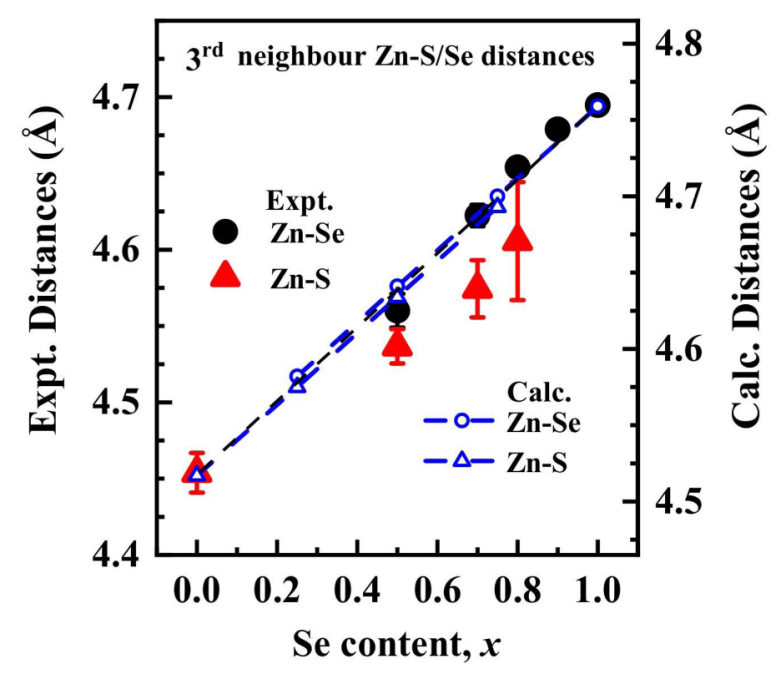

FIG. 6. Experimental estimates of third nearest-neighbor Zn-Se (black-solid circles) and $\mathrm{Zn}-\mathrm{S}$ (red-solid triangles) distances as a function of the composition $x$. The same bond distances obtained from calculations are shown with corresponding blue-open symbols.

angles. Finally, we can compare the evolution of the bond angles for the second coordination shells in cationic solid solutions (addressed in Ref. [23]) and anionic solid solutions (addressed here). In both cases, changes in the bond angles are primarily responsible for the changes in the lattice parameters. However, the cationic solid solution has been shown [23] to be controlled by changing anion-centered bond angles with the cation-centered angles relatively constant. On the contrary, the reverse is seen for anionic solid solutions, as illustrated in Fig. 5. We shall return to the discussion on the implication of this finding later in the text, after presenting the variations in the third coordination shell.

The experimental estimates of the bond lengths $\mathrm{Zn}-\mathrm{Se}$ and $\mathrm{Zn}-\mathrm{S}$ in the third coordination shell are reported in Fig. 6, with solid-black circles and red triangles, respectively. The corresponding distances from the theoretically calculated results are also shown, with corresponding blue-open symbols, in the same figure, once again in good agreement with the experimental data. As done in the previous plots, we draw a dashed-black line joining the $\mathrm{Zn}-\mathrm{S}$ and $\mathrm{Zn}-\mathrm{Se}$ bond distances in pure $\mathrm{ZnS}$ and $\mathrm{ZnSe}$, indicating the variation with composition that would be expected on the basis of Vegard's law. The other data points reported in Fig. 6 are shown to be in a remarkable agreement with this Vegard's law dictated variation. This is particularly evident for the $\mathrm{Zn}-\mathrm{Se}$ bond distances, where most of the experimental data points with very small error bars appear virtually on the dashed line. The composition dependent $\mathrm{Zn}-\mathrm{S}$ bond distances, instead, show a small deviation from the dashed line, though much smaller than the deviations seen for the first and second coordination shells. Without a doubt, a large part of this discrepancy arises from the sizable uncertainty in the estimates of the $\mathrm{Zn}-\mathrm{S}$ distances for the dilute sulphide compounds, which is also reflected by the large error bars. Keeping this uncertainty in mind, even the $\mathrm{Zn}-\mathrm{S}$ bond distance can be considered in good agreement with what is expected from Vegard's law. This conclusion is further supported by the fact that the values extracted from 


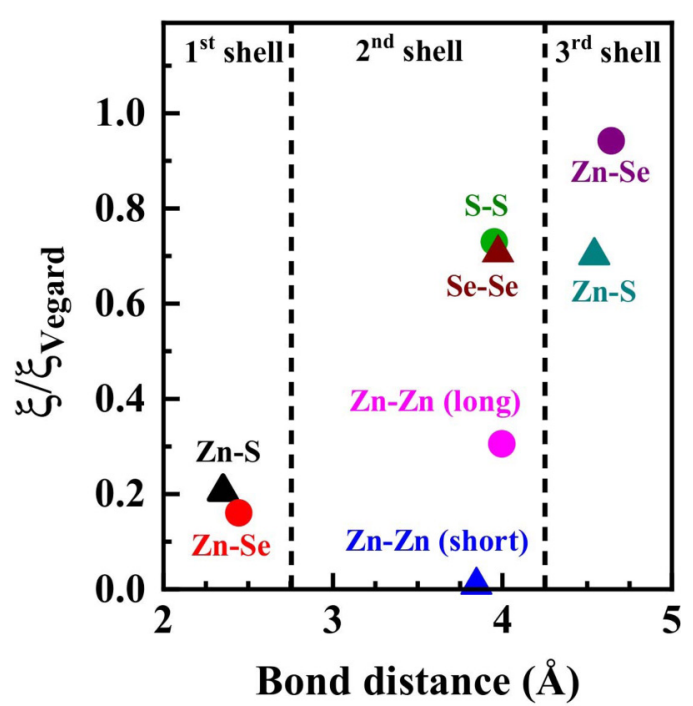

FIG. 7. Relative deviations of the rates of change of various interatomic distances with the composition from those expected from an application of Vegard's law plotted as a function of the corresponding interatomic distance, characterizing different coordination shells of the solid.

the electronic structure calculations fall exactly on top of the dashed line.

Thus, our analysis provides an understanding of the evolution of the microscopic atom-pair distances successively through first, second and third coordination shells, leading to an average description of the microscopic justification of Vegard's law for the lattice parameter variation. The individual bond distances for the first and second coordination shells differ significantly from the Vegard's law type expectation, while in the third coordination shell this expectation is fulfilled rather accurately. This suggests that the lengthscale over which the interatomic distances begin to mimic Vegard's law is approximately equal or larger than the distances found in the third coordination shell. Therefore, this is the microscopic origin of Vegard's law concerning the lattice parameters, since the lattice parameters are determined by the diffraction technique that averages over a much longer length scale. Additionally, it is extremely important to keep in mind that this conformity of the interatomic distances with the Vegard's law is brought about primarily by suitable modifications in the bond angles and not by changes in the bond lengths, underlining the difference in the structural evolutions due to compositional changes and those realized under the application of pressure.

For a more quantitative visualization of the previous reasoning, we can employ $\xi$ and $\xi_{\text {vegard, }}$, which respectively denote the calculated rate of change of a given interatomic pair distance with the composition and the corresponding rate of change expected from Vegard's law. For each bond length in each coordination shell, it is useful to evaluate the relative departure from the Vegard s law defined by $\xi / \xi_{\text {Vegard }}$.

This deviation has been plotted for different interatomic pairs in Fig. 7, as a function of the interatomic distance up to the third coordination shell. Within the experimental uncertainties, this figure provides a clear view of how the interatomic distances evolve and approach the behavior predicted by Vegard's law, given by $\xi / \xi_{\text {Vegard }}=1$. Clearly, both bond distances corresponding to the first coordination shell, i.e. $\mathrm{Zn}-\mathrm{S}$ and $\mathrm{Zn}-\mathrm{Se}$, exhibit a pronounced departure from the Vegard's law with $\xi / \xi_{\text {Vegard }} \sim 0.2$, indicating that the individual bond distances are more rigid than would be naively expected based on the evolution of the lattice parameters (or Vegard's law) as a function of the composition. We note that the deviation from the Vegard's law observed [23] earlier for closely related $\mathrm{Zn}-\mathrm{S}$ and $\mathrm{Cd}-\mathrm{S}$ first-neighbor distances in $\mathrm{Zn}_{1-x} \mathrm{Cd}_{x} \mathrm{~S}$ was somewhat more pronounced, indicating higher degrees of rigidity of these bonds, compared to what we find here for $\mathrm{Zn}-\mathrm{S}$ and $\mathrm{Zn}-\mathrm{Se}$ first-neighbor bond distances. Interestingly, the second coordination shell of the present anionic solid-solution series presents two contrasting scenarios, based on whether we consider cation-cation (metal-metal) distances or anion-anion (ligand-ligand) distances. Two distinct cation-cation distances are found in the system [see Fig. 4(a)], designated as short and long ones. Neither of these two interatomic distances show any significant variations as a function of the composition, leading to a rather small $\xi / \xi_{\text {Vegard }}(\leqslant 0.3)$. Among these two, we note that the estimates of the short $\mathrm{Zn}-\mathrm{Zn}$ bond is more error prone [see Fig. 4(a)]; therefore, the estimated $\xi / \xi_{\text {vegard }} \sim 0.3$ for the long $\mathrm{Zn}-\mathrm{Zn}$ atomic distances is a more reliable estimate. We also note that this value is in good agreement with the value of $\xi / \xi_{\text {vegard }}(0.25)$ estimated from the theoretical evaluation of the interatomic distances shown in Fig. 4(a) with blue symbols and dashed lines. Interestingly, $\mathrm{S}-\mathrm{S}$ and $\mathrm{Se}-\mathrm{Se}$ interatomic distances, still belonging to the second coordination shell, show a much more rapid variation with the composition, in closer agreement with expectations based on Vegard's law, as indicated by the large values of $\xi / \xi_{\text {vegard }} \sim 0.72$. These distinctive behaviors of rigidity in $\mathrm{Zn}-\mathrm{Zn}$ and adaptability in S-S and $\mathrm{Se}-\mathrm{Se}$ bond distances with changing lattice parameters across the solid solution can only arise from a relative rigidity of the $\mathrm{Zn}-\mathrm{S}-\mathrm{Zn}$ and $\mathrm{Zn}-\mathrm{Se}-\mathrm{Zn}$ bond angles close to the tetrahedral angle as in the pure samples, simultaneously with a flexible $\mathrm{S}-\mathrm{Zn}-\mathrm{S}$ and $\mathrm{Se}-\mathrm{Zn}-\mathrm{Se}$ bond angles. This clearly contrasts the scenario encountered in the case of the cation substituted solid solution of $\mathrm{Zn}_{1-x} \mathrm{Cd}_{x} \mathrm{~S}$ [23] where $\mathrm{Zn}-\mathrm{S}-\mathrm{Zn}$ and $\mathrm{Cd}-\mathrm{S}-\mathrm{Cd}$ bond angles were found to evolve across the composition series with a relatively inflexible $\mathrm{S}-\mathrm{Zn}-\mathrm{S}$ and $\mathrm{S}-$ $\mathrm{Cd}-\mathrm{S}$ bond angles. Figure 7 shows that the interatomic $\mathrm{Zn}-\mathrm{S}$ and $\mathrm{Zn}-\mathrm{Se}$ distances in the third coordination evolve almost in conformity with the lattice parameter variation dictated by the Vegard's law, indicated by near unity value of $\xi / \xi_{\text {Vegard }}$.

We now strive to rationalize this difference between the cationic substituted systems and the anionic substituted ones in terms of the pivotal element defining the changing bond angle to accommodate the varying composition and, hence, the microscopic origin of changes in the lattice parameters. In Ref. [23], it was originally observed that anion centered bond angles changed significantly while the cation centered ones remained rigidly close to the tetrahedral angle of the pure end members in the case of a cation-substitution $\left(M_{1-x} M_{x}^{\prime} X\right)$ series. This led to identifying two fundamental building blocks of the substituted compositions, namely rigid $M X_{4}$ and $M^{\prime} X_{4}$ units. These building blocks were connected through the corner-sharing of an $X$ atom. The potential to change the $X$ - 
centered angles was assumed to be softer than that to change the $M$-centered ones. However, this hypothesis is contradicted by our present results on anionic substituted solid solution series, where we find a relative rigidity of the $X$-centered bond angles while the $M$-centered ones remain soft. If we have to think in terms of fundamental building blocks, we shall then be forced to assume rigid $M_{4} X$ and $M_{4} X^{\prime}$ tetrahedral units for the anionic substituted systems. This indicates that neither $M_{4} X$ nor $M X_{4}$ tetrahedral units are fundamental building blocks from energetic considerations. It is easy to understand the relative invariance of the $M-X$ bond distances irrespective of the substitution type, as this is controlled by the covalency/hybridization between the two sites, $M$ and $X$; in fact, this constitutes the basic concept for defining the covalent radii of various atoms in specific oxidation states irrespective of the specific solid in which such a bond may exist. However, the interatomic distances between the same type of atoms, such as the $M-M$ and $X-X$ bond distances, are controlled relatively less by covalency and more by the requirement of minimising the electrostatic repulsions between similar atoms. This observation provides us with a clue to understanding the apparently different behaviors of the two types of substituted systems on a common platform. Assuming that the solid solution affects one sublattice, the adjustments required to minimize the electrostatic repulsions are best achieved by displacing the nearby atoms under the condition that the covalent radii in the same sublattice are separately preserved for each atomic type. This implies that, upon cationic substitution, the average cation-cation distance in the second coordination shell is expected to change, while the average anion-anion distance should remain relatively unaffected. The opposite trend is expected upon anionic substitution, and this is indeed what has been observed in the present experimental and theoretical data. Together with the relative rigidity of the $M-X$ bond lengths, this implies that the $M-X-M$ angles should change and $X-M-X$ angles should not change in $M_{1-x} M_{x}^{\prime} X$ cationic solid solutions, while the $M-X-M$ angles should not change and $X-M-X$ angles should change in $M X_{1-x} X_{x}^{\prime}$ anionic solid solutions.

[1] L. Vegard, Z. Phys. 5, 17 (1921).

[2] A. R. Denton and N. W. Ashcroft, Phys. Rev. A 43, 3161 (1991).

[3] J. Fritsch, O. F. Sankey, K. E. Schmidt, and J. B. Page, J. Phys.: Condens. Matter 11, 2351 (1999).

[4] H. L. Ray, N. Zhao, and L. C. De Jonghe, Electrochim. Acta 78, 294 (2012).

[5] J. Pellicer-Porres, A. Polian, A. Segura, V. Muñoz-Sanjosé, A. Di Cicco, and A. Traverse, J. Appl. Phys. 96, 1491 (2004).

[6] J. C. Mikkelsen and J. B. Boyce, Phys. Rev. B 28, 7130 (1983).

[7] Z. Wu, K. Lu, Y. Wang, J. Dong, H. Li, C. Li, and Z. Fang, Phys. Rev. B 48, 8694 (1993).

[8] A. Balzarotti, N. Motta, A. Kisiel, M. Zimnal-Starnawska, M. T. Czyżyk, and M. Podgórny, Phys. Rev. B 31, 7526 (1985).

[9] R. E. Nahory, M. A. Pollack, W. D. Johnston, and R. L. Barns, Appl. Phys. Lett. 33, 659 (1978).
Finally, our paper establishes that these very changes are the microscopic origin of the century-old Vegard's law, which is an average statement connecting changes in the lattice parameters to changes in the composition. Our results also underline the qualitatively dissimilar manner of the microscopic evolution of structural aspects with solid solutions, often mistakenly thought of as "chemical" pressure, in comparison to the microscopic structural changes under the application of physical pressure.

\section{ACKNOWLEDGMENT}

IISc group of authors thanks Science and Engineering Research Board, Department of Science and Technology, Government of India and Jamsetji Tata Trust for support of this research. We acknowledge DESY (Hamburg, Germany), a member of the Helmholtz Association HGF, and Elettra Sincrotrone, Trieste for the provision of experimental facilities. The financial supports for carrying out synchrotron experiments from NanoMission and the Department of Science and Technology under the Indo-Italian Program of Cooperation are gratefully acknowledged. The computational work was enabled by resources provided by the Swedish National Infrastructure for Computing (SNIC) at UPPMAX, partially funded by the Swedish Research Council through Grant Agreement No. 2018-05973. I.D.M. and S.S. acknowledge financial support from the National Research Foundation (NRF) funded by the Ministry of Science of Korea, through the Mid-Career Grant No. 2020R1A2C101217411. The work of I.D.M. is also supported by the appointment to the JRG program at the APCTP through the Science and Technology Promotion Fund and Lottery Fund of the Korean Government, as well as by the Korean Local Governments, Gyeongsangbuk-do Province and Pohang City. O.E. acknowledges financial support from Knut and Alice Wallenberg Foundation, the Swedish Research Council (VR), eSSENCE, STandUPP, the Swedish Energy Agency, the Foundation for Strategic Research (SSF), and the ERC (Synergy Grant FASTCORR, project 854843).

[10] E. Sidot, A. Kahn-Harari, E. Cesari, and L. Robbiola, Mater. Sci. Eng. A 393, 147 (2005).

[11] R. J. Heritage, P. Porteous, and B. J. Sheppard, J. Mater. Sci. 5, 709 (1970).

[12] C. Bocchi, S. Franchi, F. Germini, A. Baraldi, R. Magnanini, D. D. Salvador, M. Berti, and A. V. Drigo, J. Appl. Phys. 89, 4676 (2001).

[13] M. Castellanos and A. R. West, J. Mater. Sci. Lett. 3, 786 (1984).

[14] A. V. G. Chizmeshya, M. R. Bauer, and J. Kouvetakis, Chem. Mater. 15, 2511 (2003).

[15] D. Zhou and B. F. Usher, J. Phys. D 34, 1461 (2001).

[16] P. Ganguly, N. Shah, M. Phadke, V. Ramaswamy, and I. S. Mulla, Phys. Rev. B 47, 991 (1993).

[17] Y.-k. Kuo, B.-T. Liou, S.-H. Yen, and H.-Y. Chu, Opt. Commun. 237, 363 (2004). 
[18] B. T. Liou, S. H. Yen, and Y. K. Kuo, Appl. Phys. A 81, 651 (2005).

[19] W. Li, M. Pessa, and J. Likonen, Appl. Phys. Lett. 78, 2864 (2001).

[20] S. T. Murphy, A. Chroneos, C. Jiang, U. Schwingenschlögl, and R. W. Grimes, Phys. Rev. B 82, 073201 (2010).

[21] A. G. Thompson and J. C. Woolley, Can. J. Phys. 45, 255 (1967).

[22] J. C. Mikkelsen, Jr. and J. B. Boyce, Phys. Rev. Lett. 49, 1412 (1982).

[23] S. Mukherjee, A. Nag, V. Kocevski, P. K. Santra, M. Balasubramanian, S. Chattopadhyay, T. Shibata, F. Schaefers, J. Rusz, C. Gerard, O. Eriksson, C. U. Segre, and D. D. Sarma, Phys. Rev. B 89, 224105 (2014).

[24] S. Mukherjee, H. Ganegoda, A. Kumar, S. Pal, C. U. Segre, and D. D. Sarma, Inorg. Chem. 57, 9012 (2018).

[25] E. Welter, R. Chernikov, M. Herrmann, and R. Nemausat, AIP Conf. Proc. 2054, 040002 (2019).

[26] A. D. Cicco, G.Aquilanti, M. Minicucci, E. Principi, N. Novello, A. Cognigni, and L. Olivi, J. Phys.: Conf. Ser. 190, 012043 (2009).

[27] B. Ravel and M. Newville, J. Synchrotron Radiat. 12, 537 (2005).

[28] S. I. Zabinsky, J. J. Rehr, A. Ankudinov, R. C. Albers, and M. J. Eller, Phys. Rev. B 52, 2995 (1995).

[29] See Supplemental Material at http://link.aps.org/supplemental/ 10.1103/PhysRevB.104.184113 for a detailed discussion on the EXAFS data analysis.
[30] P. E. Blöchl, Phys. Rev. B 50, 17953 (1994).

[31] G. Kresse and J. Hafner, Phys. Rev. B 47, 558 (1993).

[32] G. Kresse and J. Hafner, Phys. Rev. B 49, 14251 (1994).

[33] G. Kresse and J. Furthmüller, Phys. Rev. B 54, 11169 (1996).

[34] G. Kresse and J. Furthmüller, Comput. Mater. Sci. 6, 15 (1996).

[35] J. P. Perdew, K. Burke, and M. Ernzerhof, Phys. Rev. Lett. 77, 3865 (1996).

[36] R. Elmer, M. Berg, L. Carlen, B. Jakobsson, B. Noren, A. Oskarsson, G. Ericsson, J. Julien, T. F. Thorsteinsen, M. Guttormsen, G. Lovhoiden, V. Bellini, E. Grosse, C. Muntz, P. Senger, and L. Westerberg, Phys. Rev. Lett. 77, 4884 (1996); 78, 1396(E) (1997).

[37] M. K. Rabadanov, A. A. Loshmanov, and Y. V. Shaldin, Crystallogr. Rep. 42, 592 (1997).

[38] A. Zunger, S.-H. Wei, L. G. Ferreira, and J. E. Bernard, Phys. Rev. Lett. 65, 353 (1990).

[39] A. Van de Walle, P. Tiwary, M. De Jong, D. Olmsted, M. Asta, A. Dick, D. Shin, Y. Wang, L.-Q. Chen, and Z.-K. Liu, Calphad 42, 13 (2013).

[40] A. Van De Walle, M. Asta, and G. Ceder, Calphad 26, 539 (2002).

[41] A. van de Walle and G. Ceder, J. Phase Equilib. 23, 348 (2002).

[42] A. Van De Walle, Calphad 33, 266 (2009).

[43] T. Chattopadhyay, H. von Schnering, W. Grosshans, and W. Holzapfel, Physica B+C 139-140, 356 (1986).

[44] L. Bellaiche and D. Vanderbilt, Phys. Rev. B 61, 7877 (2000). 